\title{
Effects of Concentric and Eccentric Training on the Stiffness and Blood Circulation of the Patellar Tendon
}

(๑)(1) $\circledast$

\author{
Authors \\ Keitaro Kubo ${ }^{1}$, Hideaki Yata² \\ Affiliations \\ 1 Department of Life Science, The University of Tokyo, Meguro, \\ Tokyo, Japan \\ 2 Department of Human and Environmental Well-being, Wako \\ University, Machida, Tokyo, Japan
}

Key words

knee extensors, patellar tendon, blood volume, oxygen saturation, human

accepted after revision 26.10 .2016

Bibliography

DOI http://dx.doi.org/10.1055/s-0042-121000

Sports Medicine International Open 2017; 1: E43-E49

(c) Georg Thieme Verlag KG Stuttgart · New York

ISSN 0000-000X

Correspondence

Keitaro Kubo, Ph.D.

Department of Life Science (Sports Sciences)

The University of Tokyo

Komaba 3-8-1

Meguro-ku
153-8902 Tokyo

Japan

Tel.: + 81/3/5454 6864, Fax: + 81/3/5454 4317

kubo@idaten.c.u-tokyo.ac.jp

\begin{abstract}
The aim of the present study was to investigate the effects of concentric (CON) and eccentric (ECC) training on stiffness and blood circulation of the tendon, and clarify whether relative changes in tendon stiffness are related to those in tendon blood circulation. 17 subjects were assigned to training $(n=9)$ and control $(n=8)$ groups. Training group completed 12 weeks of unilateral training program for knee extensors. They performed 5 sets of CON or ECC at $80 \%$ of 1 RM with 10 repetitions. Before and after training, cross-sectional area, stiffness, hysteresis, blood volume, and oxygen saturation of the patellar tendon were measured. Oxygen saturation significantly increased with CON and ECC. Stiffness significantly increased with CON but not with ECC. Other measured variables did not change after training. Furthermore, the relative change in tendon stiffness did not correlate with that in blood volume or oxygen saturation. In control group, measured variables did not change after training. These results suggest that tendon stiffness increased after CON but not after ECC; however no significant differences in the changes observed in size and blood circulation of the tendon were found between 2 protocols. Furthermore, changes in tendon stiffness were not related to those in tendon blood circulation.
\end{abstract}

\section{Introduction}

Previous studies reported reduced pain levels in patients with tendinopathy following treatments involving eccentric training $[2,10,21,25]$. However, the mechanisms responsible for the effectiveness of eccentric training currently remain unclear. Kjaer et al. [11] suggested that blood circulation within tendons contributes to their repair following various physical activities. We demonstrated that it was possible to evaluate blood circulation (blood volume and oxygen saturation) in human tendons using a laser oxygenation monitor [18]. With this technique, we found that changes in blood circulation of tendons differed among different contraction modes $[15,20]$. For example, blood volume of the patellar tendon did not change after 3 months of isometric knee extension training, but significantly increased after dynamic training [20]. We more recently showed that changes in blood circulation of the Achilles tendon during and after repeated eccentric contractions were more prominent than those during and after repeated concentric contractions [15]. Yin et al. [30] also reported increases in blood volume and oxygen saturation of the patellar tendon after repeated knee extension eccentric exercises. Therefore, blood vol- ume and oxygen saturation of tendons may be markedly higher after long-term eccentric training than concentric training.

Resistance training involving eccentric contractions has been shown to achieve greater improvements in muscle strength and size than that involving concentric contractions [1, 9]. In the last few decades, several studies have used ultrasonography to investigate the effects of various resistance training programs on the mechanical properties of human tendons in vivo $[3,20]$. However, information regarding the effects of eccentric training on tendon properties remains limited [5, 6, 22-24]. These previous studies, except for that by Malliaras et al. [23], indicated that resistance training programs involving eccentric contractions resulted in only slight increases or no changes in tendon stiffness $[5,6,22,24]$. Foure et al. [6] reported that Achilles tendon stiffness did not change after 14 weeks of eccentric training. On the other hand, Malliaras et al. [23] showed that tendon stiffness increased by $59 \%$ after eccentric training, and markedly increased $(+75 \%)$ with decreases in maximal elongation after concentric training. These findings indicate that increases in tendon stiffness after eccentric training are smaller than those after concentric training. 
In the present study, we compared changes in blood circulation and stiffness of the patellar tendon after concentric and eccentric training. We hypothesized that eccentric training produces larger increases in tendon blood volume and oxygen saturation and smaller increases in tendon stiffness than concentric training. Our previous finding demonstrated that blood volume of the patellar tendon significantly increased after 12 weeks of dynamic training (tendon stiffness slightly increased), whereas it did not change after isometric training (tendon stiffness markedly increased) [20]. Therefore, we also expected changes in tendon stiffness to be related to the changes induced in tendon blood circulation by the concentric and eccentric training protocols.

\section{Materials and Methods Subjects}

17 healthy males volunteered for this investigation. Subjects were randomly assigned to a training group $(n=9$; age: $20.8 \pm 0.5 \mathrm{yrs}$, height: $173.6 \pm 5.5 \mathrm{~cm}$, weight: $65.6 \pm 7.7 \mathrm{~kg}$, mean $\pm \mathrm{SD}$ ) and control group ( $n=8$; age: $20.5 \pm 0.4 \mathrm{yrs}$, height: $169.4 \pm 6.0 \mathrm{~cm}$, body mass: $67.6 \pm 8.7 \mathrm{~kg}$ ). Subjects were physically active but had not participated in any organized program involving regular exercise for at least 1 year before testing. They were fully informed of the procedures to be utilized as well as the purpose of the study. All subjects provided written informed consent. All procedures met the ethical standards set by the International Journal of Sports Medicine [7]. This study was approved by the Ethics Committee for Human Experiments, Department of Life Science (Sports Sciences), The University of Tokyo.

\section{Training}

Subjects performed a unilateral knee extension exercise in a seated position with a knee extension machine (Everynew, Tokyo, Japan). The range of motion of the knee joint was from $90^{\circ}$ to $0^{\circ}$ $\left(0^{\circ}=\right.$ full extension $)$. Subjects trained 3 times a week for 12 weeks. They trained one leg with concentric contractions (CON) and the other leg with eccentric contractions (ECC). In each subject, the right and left legs were randomly allocated to each of the 2 training protocols. In CON, subjects performed a concentric knee extension at a constant velocity for approximately $1 \mathrm{~s}$, and then returned to the starting position over $3 \mathrm{~s}$ with the investigator holding the weight to prevent eccentric contractions. In ECC, subjects performed an eccentric knee extension while lowering a weight at a constant velocity for approximately $3 \mathrm{~s}$, and then returned to the starting position over $1 \mathrm{~s}$ with the investigator holding the weight to prevent concentric contractions. In the present study, subjects repeated the movement at an approximately constant speed and frequency with the aid of a metronome. They performed 5 sets of exercise, which consisted of unilateral knee extensions at $80 \%$ of one repetition maximum ( $1 \mathrm{RM}$ ) with 10 repetitions per set, with a rest interval of $1 \mathrm{~min}$. In the training session, subjects trained the CON protocol leg first and then the ECC protocol leg, and in the next session the order was reversed. The measurement of 1 RM was performed every 4 weeks to adjust the training load. Throughout the experimental period, 1 RM values significantly increased by $62.4 \pm 13.9 \%$ for CON and $48.5 \pm 17.3 \%$ for ECC (both $p<0.001$, - Fig. 1), and no significant difference in the relative increase in 1 RM was found between CON and ECC $(p=0.079)$. No significant dif-

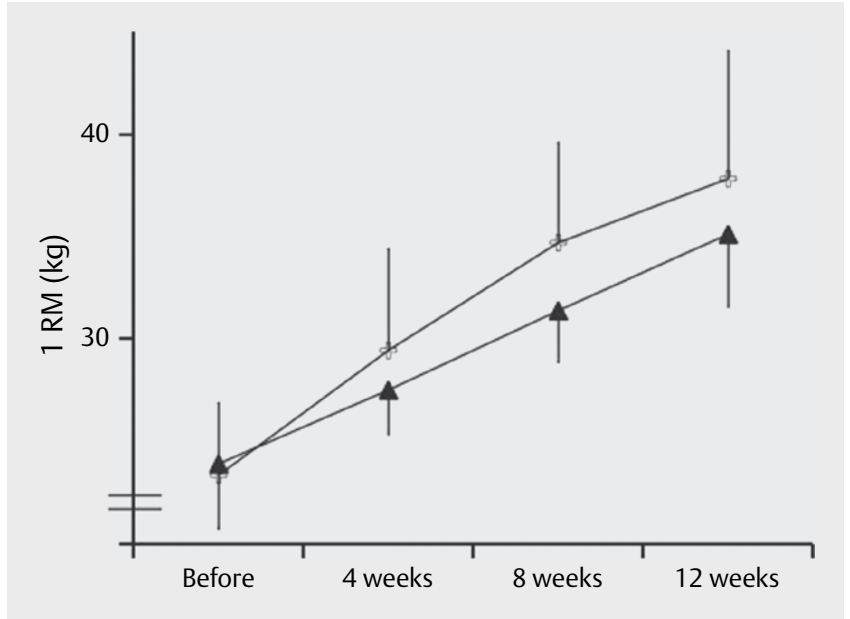

- Fig. 1 Changes in 1 RM with CON (open cross) and ECC (closed triangle) during the experimental period.

ferences were noted in 1 RM before and after 4,8 , and 12 weeks between CON and ECC. In the present study, the mean 1 RM of both legs was adopted to make the load of each leg equal.

\section{Experimental design}

Before the experimental phase of this study was initiated, subjects visited the laboratory in order to become familiar with the measurements of muscle and tendon properties as described below. All subjects were tested at baseline (before training) and 3-7 days after the last training session (after training). All measurements were performed at almost the same time of day in order to minimize diurnal effects. In the training group, the right and left legs of each subject were measured on different days in order to avoid the effects of fatigue. In the control group, only the right legs of subjects were measured.

\section{Tendon cross-sectional area}

The cross-sectional area of the patellar tendon was measured using an ultrasonic apparatus (SSD-6500, Aloka, Tokyo, Japan) at $50 \%$ of the patellar tendon length. Subjects remained in a supine position with the knee joint flexed at an angle of $90^{\circ}$ (full extension $=0^{\circ}$ ) and the muscles relaxed. An outline of the tendon was traced from the axial image, and the traced image was transferred to a computer in order to calculate the tendon CSA using open-source image analysis software (Image J, NIH, Bethesda, MD).

The repeatability of the measurement of tendon cross-sectional area was investigated on 2 separate days in a preliminary study with 7 young males. No significant differences were observed between the test and retest values of the tendon cross-sectional area. The intraclass correlation coefficient (ICC) and coefficient of variation (CV) were 0.92 and $2.7 \%$, respectively.

\section{Blood volume and oxygen saturation of patellar tendon}

The posture of subjects was the same as that for the measurement of the tendon cross-sectional area described above. During the 
resting state, we measured blood circulation (oxyhemoglobin; Oxy, deoxyhemoglobin; Deoxy, total hemoglobin; THb, oxygen saturation; $\mathrm{StO}_{2}$ ) of the patellar tendon. In order to measure blood circulation of the tendon using a laser oxygenation monitor (BOML1TRSF, Omega Wave), a probe was positioned at the middle site of the patellar tendon. This instrument uses 3 red laser lights (635, 650 , and $690 \mathrm{~nm}$ ) and calculates the relative tissue levels of Oxy, Deoxy, and THb (corresponding to blood volume) according to the Beer-Lambert law. The distance between the light source and photodetector was $8 \mathrm{~mm}$. The measured variables at a specific depth (measurement depth of $5-8 \mathrm{~mm}$ ) of the tissue were measured by changing the location of the 2 detectors $[12,16]$. Details of the technique and principles of this instrument (red laser lights) have been described in our previous studies [16, 18]. Briefly, the 2-point detection and differential calculation methods were used to measure blood circulation in the deep region of the tissue only (see - Fig. 1 of Ref 16). Oxy, Deoxy, and THb at specific tissue depths were measured by changing the location of the 2 detectors. The offset values of Oxy, Deoxy, and THb were reduced, and a highly sensitive measurement was achieved using the 2-point detection method. The precision and validity of this procedure were presented and discussed in our previous studies $[16,18]$. In the present study, data obtained at the superficial site (measurement depth of $0-5 \mathrm{~mm}$; including the skin and subcutaneous fat) were also presented.

In the present study, the units of Oxy, Deoxy, and THb were expressed as $\mu \mathrm{mol} / \mathrm{l}$, although this did not represent the actual physical volume. $\mathrm{StO}_{2}$ was calculated from Oxy and $\mathrm{THb}$ values using the following formula: $\mathrm{StO}_{2}(\%)=100 * \mathrm{Oxy} \cdot \mathrm{THb}^{-1}$. Data were input into a personal computer at a sampling frequency of $10 \mathrm{~Hz}$ via an A/D transducer (Power Lab, AD Instruments, Australia). Mean values over a given duration (approximately $5 \mathrm{~min}$ ) were calculated using analytical software (Chart ver. 7.1, AD Instruments, Australia). On each measurement day of the right and left legs, blood circulation was measured in both the right and left patellar tendons. The measured values that are shown below are the means of 2 tests.

The repeatability of measurements of tendon $\mathrm{THb}_{\mathrm{b}}$ and $\mathrm{StO}_{2}$ was confirmed in our previous studies $[18,20]$. In the present study, comparisons of $\mathrm{THb}$ and $\mathrm{StO}_{2}$ values of the patellar tendon between the 2 tests (on each day of the measurements for the right and left legs) revealed no significant differences, and ICC and CV were 0.82 and $5.0 \%$ for $\mathrm{THb}$ and 0.84 and $5.7 \%$ for $\mathrm{StO}_{2}$, respectively.

\section{Mechanical properties of the patellar tendon}

A subject sat in a specially designed isometric dynamometer (Applied Office, Tokyo, Japan) with support for the back, and the hip joint was flexed at an angle of $80^{\circ}$ (full extension $=0^{\circ}$ ) to standardize measurements and localize the action to the appropriate muscle group. The ankle was firmly attached to the lever arm of the dynamometer with a strap and fixed with the knee joint flexed at an angle of $90^{\circ}$. The center of rotation of the dynamometer was visually aligned with the center of rotation of the knee joint under submaximal contraction conditions. Prior to testing, each subject performed a standardized warm-up and submaximal contractions to become accustomed to the test procedure. Subjects were instructed to develop a gradually increasing force from a relaxed state to a maximal voluntary contraction (MVC) within $5 \mathrm{~s}$, followed by grad- ual relaxation within $5 \mathrm{~s}$. The task was repeated 2 times per subject with at least 3 min between trials. The measured values that are shown below are the means of 2 trials.

The measured knee joint torque (TQ) was converted to tendon force by the following equation:

Tendon force $=\mathrm{TQ} \cdot \mathrm{MA}^{-1}$

where MA is the moment arm length of the patellar tendon at $90^{\circ}$ of knee flexion (42 mm; [26]).

An ultrasonic apparatus with an $80 \mathrm{~mm}$ long probe (UST-5712, Aloka, Tokyo, Japan) was used to obtain longitudinal ultrasonic images of the patellar tendon. In the present study, elongation of the patellar tendon was assessed as a change in the distance between the tibia and distal apex of the patellar. Tendon force - elongation curves above $50 \%$ MVC were fit to a linear regression equation, the slope of which was adopted as an index of stiffness of patellar tendon. The area within tendon force - elongation loop, as a percentage of the area beneath the curve during the ascending phase, was calculated as hysteresis.

The repeatability of the measurements of tendon stiffness and hysteresis was investigated on 2 separate days in a preliminary study with 7 young males. No significant differences were observed between the test and retest values of tendon stiffness and hysteresis. ICC and CV were 0.86 and $7.9 \%$ for stiffness and 0.81 and $10.8 \%$ for hysteresis, respectively.

\section{Statistical analysis}

Values are reported as means \pm standard deviation. Differences in the tendon elongation values of tendon force-elongation curves between before and after training were analyzed by a paired Student's t-test. In the training group, differences in time (before and after training) and mode (CON, ECC, and no training) were tested using a 2-way ANOVA with repeated measures $\{3$ (modes) $\times 2$ (test times) \}. The $\mathrm{F}$ ratios for main effects and interactions were considered significant. Significant differences among means were detected using the Bonferroni post-hoc test. When ANOVA revealed significant main effects for mode and time, whether or not there was a significant interaction between them, we returned to a one-way ANOVA with repeated measures to detect any significant changes from before training. In all ANOVAs, Mauchly's sphericity test was performed to assess the homogeneity of variance. The Greenhouse-Geisser correction was applied where assumption of sphericity was violated. Spearman's rank correlation test was used to assess relationships among the measured parameters. Statistical computations were performed using IBM SPSS Statistics (version 19). Significance was set at $p<0.05$.

\section{Results}

- Fig. 2 shows the relationships between force and elongation of the patellar tendon before and after training. No significant differences were observed in tendon elongation values at any force production levels before and after training with CON and ECC. MVC values significantly increased by $17.3 \%$ with $\operatorname{CON}(p=0.002)$ and $15.2 \%$ with ECC $(p=0.026)$. Maximal elongation, hysteresis, and cross-sectional area of the patellar tendon did not change after training with CON or ECC ( $\vee$ Table 1 ). The effects of mode and the interaction between time and mode were not significant for ten- 
don stiffness, although the effect of time was significant. Stiffness of the patellar tendon significantly increased by $55.8 \%$ with CON $(p=0.014)$, but not with ECC $(p=0.246)$.
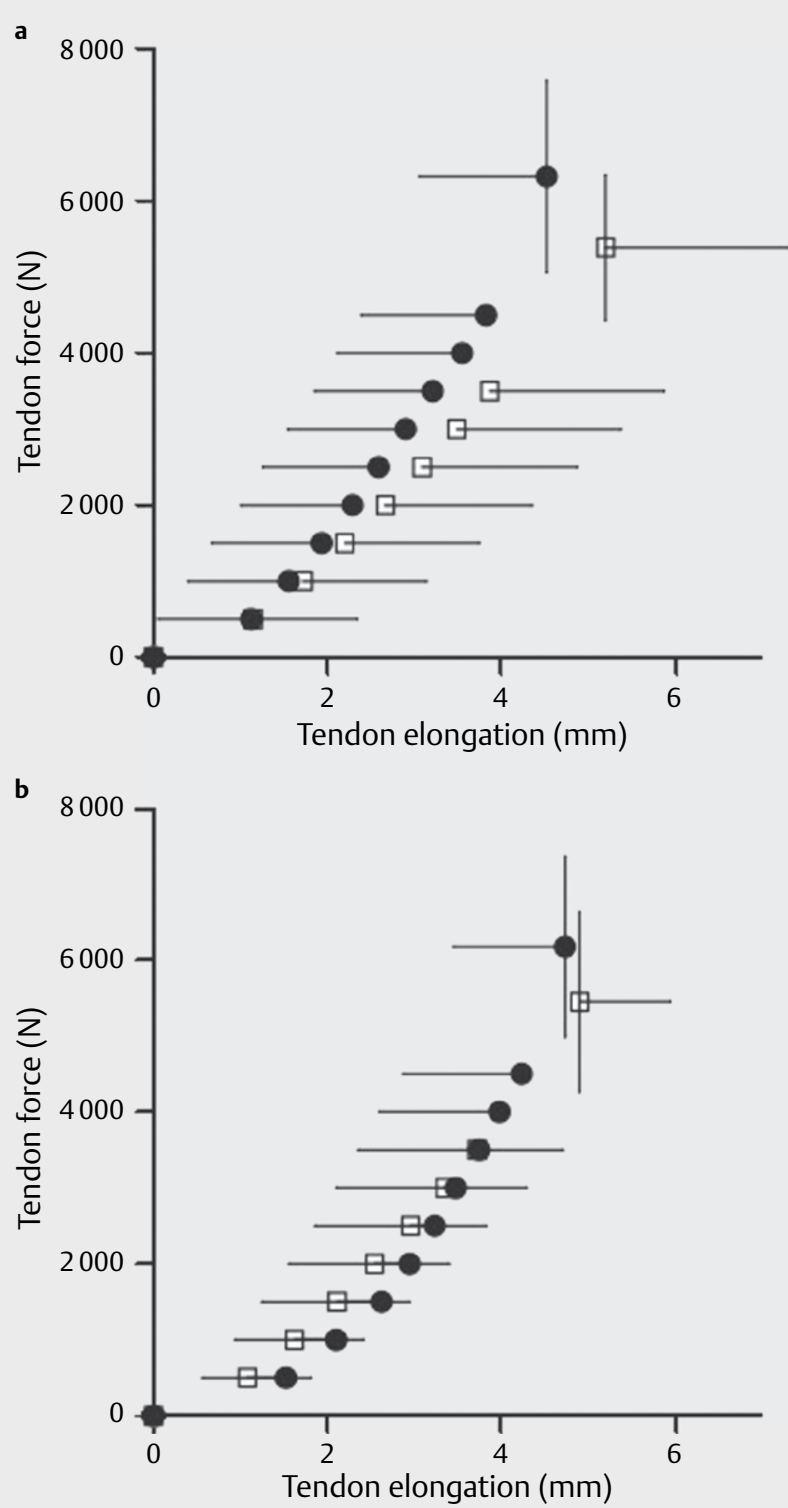

- Fig. 2 Tendon force - elongation of the patellar tendon before (open) and after (closed) training with CON a and ECC $\mathbf{b}$.
THb of the patellar tendon did not change after training with CON or ECC ( $p=0.114$ for CON, $p=0.415$ for ECC) ( $>$ Table 2$)$. Regarding $\mathrm{StO}_{2}$ of the patellar tendon, the effects of mode and the interaction between time and mode were not significant, although the effect of time was significant. $\mathrm{StO}_{2}$ of the patellar tendon increased by $8.3 \%$ with CON ( $p=0.007$ ) and by $6.3 \%$ with ECC $(p=0.095)$. THb and $\mathrm{StO}_{2}$ at the superficial site (measurement depth of $0-5 \mathrm{~mm}$ ) did not change after training with CON or ECC ( Table 2).

The relative change in tendon stiffness did not correlate with relative changes in $\mathrm{THb}(r=0.071, p=0.779)$ or $\mathrm{StO}_{2}(r=-0.028$, $\mathrm{p}=0.913$ ) of the patellar tendon ( $\vee$ Fig. 3 ).

In the control group, no significant changes were found in the measured muscle and tendon variables after training ( $\triangleright$ Table 1, 2).

\section{Discussion}

The main results of the present study were that tendon stiffness significantly increased after CON, but not after ECC. Furthermore, the changes observed in tendon stiffness were not related to changes in blood volume or oxygen saturation of the tendon.

We recently reported that changes in blood circulation of the Achilles tendon during and after repeated eccentric contractions were more prominent than those during and after repeated concentric contractions [15]. Based on this finding, we hypothesized that, after 12 weeks of training, the eccentric training protocol would produce considerable increases in tendon blood volume and oxygen saturation than the concentric training protocol. However, this hypothesis was rejected in the present study. Furthermore, we previously reported that tendon blood volume significantly increased by $47 \%$ after dynamic training (including concentric and eccentric contractions) [20]. Although the reasons for these discrepancies currently remain unknown, there are 3 possible explanations. Differences between the present results and our previous finding [15] may be due to differences in the exerted force level between the 2 protocols. In the present study, we used an identical load in order to equalize the imposed load between CON and ECC protocols. In our previous study [15], the exerted torque values during repeated eccentric contractions were approximately $20 \%$ greater than those during repeated concentric contractions. Another possible reason for the difference between our previous finding [20] and present results is the frequency of training per week (4 times per week in [20], 3 times per week in the present study). In addition, the exerted muscle force was imposed on the tendon

- Table 1 Morphological and mechanical properties of the muscle and tendon for CON, ECC, and control group Mean (sd).

\begin{tabular}{|l|c|c|c|c|c|c|}
\hline & \multicolumn{2}{|c|}{ CON } & \multicolumn{2}{c|}{ ECC } & \multicolumn{2}{c|}{ Control group } \\
\hline & Before & After & Before & After & Before & After \\
\hline MVC $(\mathrm{Nm})$ & $226(40)$ & $266(53) * *$ & $228(50)$ & $259(50)^{*}$ & $225(49)$ & $216(51)$ \\
\hline Maximal tendon elongation $(\mathrm{mm})$ & $5.2(2.1)$ & $4.5(1.5)$ & $4.9(1.0)$ & $4.7(1.3)$ & $5.1(1.0)$ & $4.7(1.1)$ \\
\hline Tendon stiffness $\left(\mathrm{N} \cdot \mathrm{mm}^{-1}\right)$ & $1004(384)$ & $1414(355) *$ & $1289(544)$ & $1457(440)$ & $1192(397)$ & $1155(385)$ \\
\hline Tendon hysteresis $(\%)$ & $20.8(11.5)$ & $18.5(7.9)$ & $20.7(10.8)$ & $18.6(9.2)$ & $23.1(9.4)$ & $23.8(9.1)$ \\
\hline Tendon cross-sectional area $\left(\mathrm{mm}^{2}\right)$ & $72.0(10.8)$ & $72.9(9.5)$ & $73.5(7.7)$ & $73.8(8.9)$ & $70.3(8.6)$ & $69.0(9.3)$ \\
\hline $\begin{array}{l}* \\
\text { maximum voluntary contraction }\end{array}$ & $*$ significantly different from before $(\mathrm{p}<0.05, \mathrm{p}<0.01$, respectively); CON, concentric training group; ECC, eccentric training group; MVC, \\
\hline
\end{tabular}


- Table 2 Blood volume and oxygen saturation of the patellar tendon and skin for CON, ECC, and control group Mean (sd).

\begin{tabular}{|l|l|l|l|l|l|l|}
\hline & \multicolumn{2}{|c|}{ CON } & \multicolumn{2}{c|}{ ECC } & \multicolumn{2}{c|}{ Control group } \\
\hline & Before & After & Before & After & Before & After \\
\hline Tendon blood volume $\left(\mu \mathrm{mol} \cdot \mathrm{I}^{-1}\right)$ & $11.7(5.1)$ & $12.5(4.1)$ & $12.1(3.0)$ & $12.9(2.4)$ & $11.3(2.6)$ & $11.6(1.9)$ \\
\hline Tendon oxygen saturation $(\%)$ & $65.5(5.5)$ & $70.6(3.2) * *$ & $66.9(6.9)$ & $70.6(4.0)$ & $67.0(6.3)$ & $68.1(4.2)$ \\
\hline Blood volume at the superficial site $\left(\mu \mathrm{mol} \cdot \mathrm{I}^{-1}\right)$ & $18.8(2.3)$ & $19.3(2.0)$ & $19.9(2.5)$ & $19.8(2.5)$ & $18.9(1.7)$ & $19.3(2.8)$ \\
\hline Oxygen saturation at the superficial site $(\%)$ & $69.6(1.9)$ & $69.7(1.2)$ & $70.6(2.4)$ & $69.7(1.2)$ & $69.8(1.5)$ & $70.2(1.3)$ \\
\hline
\end{tabular}

during either the concentric or eccentric phase only in the present study. In Kubo et al. [20], the exerted muscle force was imposed on the tendon during both contraction phases (concentric and eccentric phases). Therefore, the slight increase observed in blood volume for ECC (and CON) may be related to the lower amount of the load during training in the present study than in our previous studies $[15,20]$.

Although blood volume of the tendon did not change, oxygen saturation of the tendon increased with $\operatorname{CON}(p=0.007)$ and ECC $(p=0.095)$. In our previous study [20], oxygen saturation as well as blood volume of tendon significantly increased by $12.4 \%(p=0.033)$ (data not shown in [20]). These findings may be related to an increase in blood inflow to the tendon induced by repeated muscle contractions during the experimental period and lower oxygen consumption by the tendon $[4,17]$. On the other hand, oxygen saturation (as well as blood volume) of tendon did not change after 12 weeks of isometric training [20]. Therefore, it is clear that oxygen saturation of the tendon was increased by dynamic (regardless of the contraction mode) training. However, previous studies have indicated that treatments involving eccentric training achieved better results for tendon injuries than those involving concentric training $[10,21,25,31]$. On the other hand, other studies showed that isometric exercise and heavy slow resistance training offered clinical gains for tendinopathy $[13,29]$. Taking this point into account together with our previous findings and present results, it is possible that the effectiveness of eccentric training on chronic tendinosis may be related to other factors (e. g., changes in the mechanical properties of muscles and tendons) as well as changes in blood circulation within the tendon. For example, Mahienu et al. [22] showed that the dorsiflexion range of motion increased and passive resistive torque (corresponding to passive muscle stiffness) of plantar flexors decreased after 6 weeks of an eccentric training program, whereas Achilles tendon stiffness did not change. In future studies, we need to investigate the effects of eccentric training from various angles in order to clarify the mechanisms underlying its effectiveness.

Another interesting result of the present study was that tendon stiffness increased with CON but not with ECC; however, the difference observed in the relative increase in tendon stiffness was not significant due to the small sample size $(n=9)$. Our previous study showed that tendon stiffness markedly increased $(+83 \%)$ after isometric training but not after dynamic (including concentric and eccentric contractions) training [20]. Based on the present results and our previous findings, a slight increase in tendon stiffness after dynamic training may be largely affected by eccentric contractions during dynamic training. The present results showing the lack of changes in tendon stiffness for ECC were consistent with previous findings [5, 6, 22, 24]. On the other hand, Malliara et al. [23] reported that stiffness of the patellar tendon increased by $59 \%$ after 12 weeks of eccentric training (similar to the protocol used in the present study). In Malliara et al. [23], subjects in the eccentric training group lifted a weight with both legs and performed the lowering phase with one leg, and vice versa. Therefore, these procedures with each contraction mode group were not pure and may have been influenced by some other contraction mode. Furthermore, they showed that, after concentric training, tendon stiffness markedly increased ( $+75 \%$ ) and maximal elongation decreased. Considering these previous findings and the present results, increases in tendon stiffness by eccentric training appear to be lower than those by other contraction modes.

It currently remains unclear why the resistance training program involving eccentric contractions resulted in a slight increase or no change in tendon stiffness. Our previous study showed that blood volume of tendons significantly increased after 12 weeks of dynamic training (tendon stiffness slightly increased), whereas it did not change after isometric training (tendon stiffness markedly increased) [20]. Furthermore, we demonstrated that changes in blood circulation of the Achilles tendon during and after repeated eccentric contractions were more remarkable than those during and after repeated concentric contractions [15]. Based on these findings, we expected tendon extensibility (elasticity) to be preserved due to increases in blood volume and oxygen saturation within the tendon during the repair process after eccentric training. However, this hypothesis was rejected in the present study. In the present study, the blood volume of the tendon did not increase after eccentric training ( $\vee$ Table 2 ), and no correlations were found between relative changes in stiffness and blood volume or oxygen saturation of tendon ( $\vee$ Fig. $\mathbf{3}$ ). When these points are considered together with the present results, the slight increase observed in tendon stiffness after eccentric training may be related to other factors (e. g., neuromuscular and mechanical factors) as well as changes in tendon blood circulation. Previous studies demonstrated that, during ankle bending exercises, the ground reaction force fluctuated at a higher frequency (approximately $10 \mathrm{~Hz}$ ) during the heel-lowering (eccentric) phase $[8,27]$. Rees et al. [28] suggested that these fluctuations in force provide an important stimulus for the remodeling of injured tendons. In any case, a slight increase in tendon stiffness after eccentric training may contribute to the good clinical results achieved by treatments involving eccentric training $[2,10,21,25]$; however, the relationship between tendon stiffness and the probability of tendon injuries has not yet been proven. 


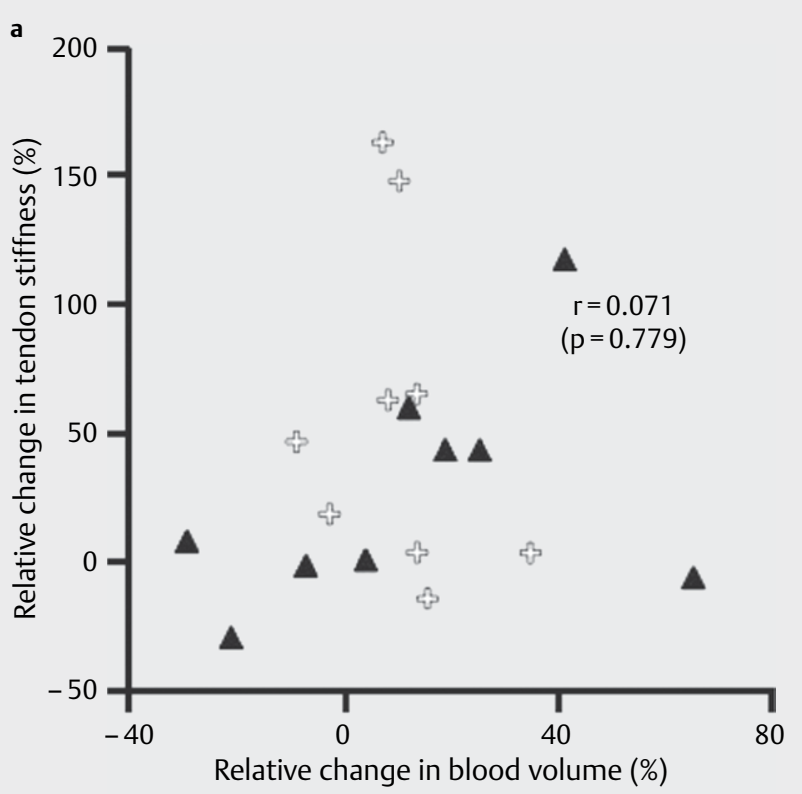

b

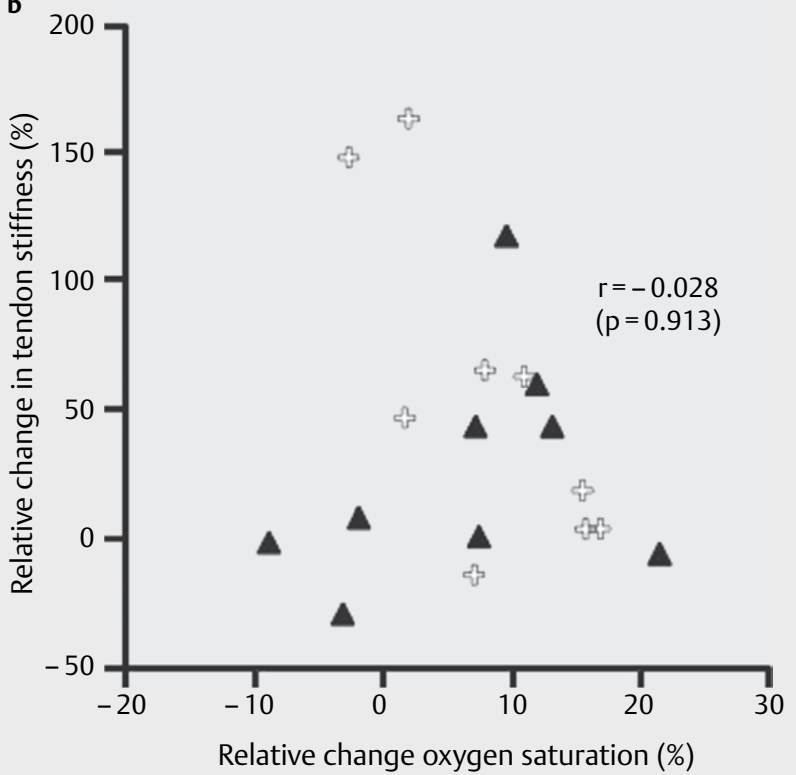

- Fig. 3 Relationships between relative changes in tendon stiffness and blood volume $\mathbf{a}$ and oxygen saturation $\mathbf{b}$ of the patellar tendon with CON (open cross) and ECC (closed triangle).

In the present study, we must draw attention to limitations and assumptions associated with the methodology followed. Firstly, an important methodological issue in the present study was whether measured $\mathrm{THb}$ and $\mathrm{StO}_{2}$ truly reflected blood circulation of the patellar tendon. We observed, using ultrasonography, that the depth of the patellar tendon from the skin was $5.1 \pm 0.6 \mathrm{~mm}$ at its superficial surface and $9.2 \pm 0.6 \mathrm{~mm}$ at its deep surface. These values remained almost unchanged after 12 weeks of training. Therefore, we confirmed that the depth of the patellar tendon from the skin was consistent with the penetration depth of red laser lights. Secondly, we measured blood circulation of the tendon at the middle site of the patellar tendon. Kongsgaard et al. [14] reported that the cross-sectional area of the patellar tendon at the proximal and distal sites significantly increased after 12 weeks resistance training but was unchanged at the middle site. Therefore, blood circulation adaptations may have occurred at the proximal and distal sites of the patellar tendon. However, we were unable to measure blood circulation of the patellar tendon at the proximal and distal sites correctly because the depth of the patellar tendon from the skin at these sites differed from that at the middle site. Thirdly, we measured blood circulation of the tendon during rest but not during exercise. In the present study, we evaluated the chronic effects of training on blood circulation of tendon. We expected acute changes (increases) in the blood circulation of the tendon to accumulate during the experimental period (12 weeks). Indeed, we previously reported that the blood volume of the tendon transiently increased after cyclic contractions [19], and thus the blood volume of the tendon during rest significantly increased after 12 weeks of resistance training (repeated cyclic contractions) [20]. Fourthly, we used an identical "load" to equalize the imposed load on the tendons between the CON and ECC protocols. However, it is very difficult to achieve the same duration during contractions between CON and ECC. Actually, subjects were unable to perform a concentric knee extension for $3 \mathrm{~s}$. Therefore, the duration of the contraction for ECC was longer than that for CON in the present study. According to the result obtained on tendon stiffness, tendon stiffness increased with CON (the contraction duration was shorter), but not with ECC (the contraction duration was longer). Therefore, this point ( $1 \mathrm{~s}$ for CON and $3 \mathrm{~s}$ for ECC) did not appear to affect the results on changes in tendon stiffness (tendon stiffness significantly increased with CON, but not with ECC).

In conclusion, tendon stiffness increased after concentric training but not after eccentric training; however, no significant differences in changes observed in size and blood circulation of the tendon were found between the 2 protocols. Furthermore, changes in tendon stiffness did not correlate with changes in blood circulation of the tendon. These results imply that the good clinical results achieved with eccentric training are related to other factors as well as changes in blood circulation within the tendon.

\section{Acknowledgements}

This study was supported by a Grant-in-Aid for Scientific Research (B) (26262173 to K. Kubo) from the Japan Society for the Promotion of Science. The authors would like to thank the staff at the Training center of Wako University for their conscientious work on this project and the subjects who participated in this study.

\section{Conflict of interest}

There is no conflict of interest with this work. 


\section{References}

[1] Aagaard P, Simonsen EB, Andersen JL, Magnusson SP, Halkjaer-Kristensen J, Dyhre-Poulsen P. Neural inhibition during maximal eccentric and concentric quadriceps contraction: effects of resistance training. J Appl Phyiol 2000; 89: 2249-2257

[2] Alfredson H, Pietila T, Jonsson P, Lorentzon R. Heavy-load eccentric calf muscle training for the treatment of chronic Achilles tendinosis. Am J Sports Med 1998; 26: 360-366

[3] Arampatzis A, Karamanidis K, Albracht K. Adaptational responses of the human Achilles tendon by modulation of the applied cyclic strain magnitude. J Exp Biol 2007; 210: 2743-2753

[4] Boushel R, Langberg H, Green S, Skovgaard D, Bulow J, Kjaer M. Blood flow and oxygenation in peritendinous tissue and calf muscle during dynamic exercise in humans. J Physiol 2000; 524: 305-313

[5] Duclay J, Martin A, Duclay A, Cometti G, Pousson M. Behavior of fascicles and the myotendinous junction of human medial gastrocnemius following eccentric strength training. Muscle \& Nerve 2009; 39 : 819-827

[6] Foure A, Nordez A, Cornu C. Effects of eccentric training on mechanical properties of the plantar flexor muscle-tendon complex. J Appl Physiol 2013; 114: 523-537

[7] Harriss D], Atkinson G. Update - ethical standards in sports and exercise science research. Int J Sports Med 2015; 36: 1121-1124

[8] Henriksen M, Aaboe J, Bliddal H, Langberg H. Biomechanical characteristics of the eccentric Achilles tendon exercise. J Biomech 2009; 42: 2702-2707

[9] Hortobagyi T, Barrier J, Beard D, Braspenninex J, Koens P, Devita P, Dempsey L, Lambert J. Greater initial adaptations to submaximal muscle lengthening than maximal shortening. J Appl Physiol 1996; 81: 1677-1682

[10] Jonsson P, Alfredson H. Superior results with eccentric compared to concentric quadriceps training in patients with jumper's knee: a prospective randomised study. Br J Sports Med 2005; 39: 847-850

[11] Kjaer M, Langberg H, Miller BF, Boushel R, Crameri R, Koskinen S, Heinemeier K, Olesen JL, Dossing S, Hansen M, Pederson SG, Rennie MJ, Magnusson P. Metabolic activity and collagen turnover in human tendon in response to physical activity. J Musculoskelet Neuronal Interact 2005; 5: 41-52

[12] Kashima S. Spectroscopic measurement of blood volume and its oxygenation in a small volume of tissue using red laser lights and differential calculation between two point detections. Opt Laser Technol 2003; 35: 485-489

[13] Kongsgaard M, Kovanen V, Aagaard P, Doessing S, Hansen P, Lausen AH, Kaldau NC, Kjaer M, Magnusson SP. Corticosteroid injections, eccentric decline squat training and heavy slow resistance training in patellar tendinopathy. Scand J Med Sci Sports 2009; 19: 790-802

[14] Kongsgaard M, Reitelseder S, Pedersen TG, Holm L, Aagaard P, Kjaer M, Magnusson SP. Region specific patellar tendon hypertrophy in humans following resistance training. Acta Physiol 2007; 191: 111-121

[15] Kubo K. Effects of repeated concentric and eccentric contractions on blood circulation of tendon. Int J Sports Med 2015; 36: 481-484

[16] Kubo K, Ikebukuro T. Blood circulation of patellar and Achilles tendons during contractions and heating. Med Sci Sports Exer 2012; 44: 2111-2117
[17] Kubo K, Ikebukuro T, Tsunoda N, Kanehisa H. Changes in oxygen consumption of human muscle and tendon following repeat muscle contractions. Eur J Appl Physiol 2008; 104: 859-866

[18] Kubo K, Ikebukuro T, Tsunoda N, Kanehisa H. Noninvasive measures of blood volume and oxygen saturation of human Achilles tendon by red laser lights. Acta Physiol 2008; 193: 257-264

[19] Kubo K, Ikebukuro T, Yaeshima K, Kanehisa H. Effects of different duration contractions on elasticity, blood volume, and oxygen saturation of human tendon in vivo. Eur J Appl Physiol 2009; 106 445-455

[20] Kubo K, Ikebukuro T, Yaeshima K, Yata H, Tsunoda N, Kanehisa H. Effects of static and dynamic training on the stiffness and blood volume of tendon in vivo. J Appl Physiol 2009; 106: 412-417

[21] Mafi N, Lorentzon R, Alfredson H. Superior short-term results with eccentric calf muscle training compared to concentric training in a randomized prospective multicenter study on patients with chronic Achilles tendinosis. Knee Surg Sports Traumatol Arthrosc 2001; 9: 42-47

[22] Mahieu NN, McNair P, Cools A, D'Haen C, Vandermeulen K, Witvrouw E. Effect of eccentric training on the plantar flexor muscle-tendon tissue properties. Med Sci Sports Exer 2008; 40: 117-123

[23] Malliaras P, Kamal B, Nowell A, Farley T, Dhamu H, Simpson V, Morrissey D, Langberg H, Maffulli N, Reeves ND. Patellar tendon adaptation in relation to load-intensity and contraction type. J Biomech 2013; 46: 1893-1899

[24] Morrissey D, Roskilly A, Twycross-Lewis R, Isinkaye T, Screen H, Woledge $R$, Bader $D$. The effect of eccentric and concentric calf muscle training on Achilles tendon stiffness. Clin Reh 2011; 25: 238-247

[25] Niesen-Vertommen SL, Taunton JE, Clement DB, Mosher RE. The effect of eccentric versus concentric exercise in the management of Achilles tendonitis. Clin J Sports Med 1992; 2: 109-113

[26] O’Brien TD, Reeves ND, Baltzopoulos V, Jones DA, Maganaris CN. Mechanical properties of the patellar tendon in adults and children. J Biomech 2010; 43: 1190-1195

[27] Rees JD, Lichtwark GA, Wolman RL, Wilson AM. The mechanism for efficacy of eccentric loading in Achilles tendon injury; an in vivo study in humans. Rheumatology 2008; 47: 1493-1497

[28] Rees JD, Wolman RL, Wilson A. Eccentric exercises; why do they work, what are the problems and how can we improve them? $\mathrm{Br}$ J Sports Med 2009; 43: 242-246

[29] Rio E, Kidgell D, Purdam C, Gaida ], Moseley GL, Pearce A], Cook ]. Isometric exercise induces analgesia and reduces inhibition in patellar tendinopathy. $\mathrm{Br}$ ] Sports Med 2015; 49: 1277-1283

[30] Yin NH, Chen WS, Wu YT, Shin TT, Rolf C, Wang HK. Increased patellar tendon microcirculation and reduction of tendon stiffness following knee extension eccentric exercises. J Orthop Sports Phys Ther 2014; 44: 304-312

[31] Young MA, Cook JL, Purdam CR, Kiss ZS, Alfredson H. Eccentric decline squat protocol offers superior results at 12 months compared with traditional eccentric protocol for patellar tendinopathy in volleyball players. Br J Sports Med 2005; 39: 102-105 